\title{
Multi Component Reactions under Increased Pressure: On the Mechanism of Formation of Pyridazino[5,4,3-de][1,6]naphthyridine Derivatives by the Reaction of Malononitrile, Aldehydes and 2-Oxoglyoxalarylhydrazones in Q-Tubes
}

\author{
Majdah A. AL-Johani ${ }^{1}$, Khadijah M. Al-Zaydi ${ }^{1, *}$, Sameera M. Mousally ${ }^{1}$, Norah F. Alqahtani ${ }^{1}$, \\ Noha Hilmy Elnagdi ${ }^{2}$ and Mohamed H. Elnagdi ${ }^{3}$ \\ 1 Department of Chemistry, Faculty of Sciences-AL Faisaliah, King Abdulaziz University, Jeddah, \\ P.O. Box 50918, Jeddah 21533, Saudi Arabia; moon98.1@hotmail.com (M.A.A.); \\ smousally@kau.edu.sa (S.M.M.); Nalqahtani00123@kau.edu.sa (N.F.A.) \\ 2 Department of Organic Chemistry, Faculty of Pharmacy, Modern University for Technology and Information, \\ Cairo, P.O. Box 12518, Cairo 11511, Egypt; elnagdinoha@yahoo.com \\ 3 Faculty of Science, Cairo University, Cairo, P.O. Box 12613, Cairo 11511, Egypt; m.h.elnagdi@outlook.com \\ * Correspondence: alzaydi_kh@yahoo.com; Tel.: +966-505-678719
}

Received: 16 October 2017; Accepted: 29 November 2017; Published: 1 December 2017

\begin{abstract}
Efficient synthesis of phenanthridin-6(5H)-one derivatives 12a-n in a four-component reaction of aldehyde hydrazone, aromatic aldehydes and malononitrile in Q-Tubes is reported. The results showed that the methodology has the advantage of being a one-pot synthesis of tricyclic systems in good yields. Potential routes leading to formation of compounds $\mathbf{1 2}$ are discussed. The structures of the synthesized compounds could be unequivocally established via X-ray crystal structure determination and spectroscopic methods.
\end{abstract}

Keywords: X-ray crystallography; arylhydrazonals; 2-amino-1,1,3-propenetricarbonitrile; pyridazines; negative activation volume

\section{Introduction}

The considerable biological and medicinal activities of pyridazines has stimulated considerable research on efficient syntheses of these derivatives in past years [1-4]. Elnagdi et al. reported synthesis of 2-amino-1,4-dihydropyridazine, an isoelectronic derivative of 1,4-dihydropyrimidines of established biological activities [5-8], via $3+3$ atom combination of arylhydrazones $1 \mathbf{a}$ and $\alpha, \beta$-unsaturated nitriles 2 [9] or by reacting a mixture of $\mathbf{1}, \mathbf{4}$ and $\mathbf{5}$ in one pot (Scheme 1). Subsequent studies [10,11] on this novel route revealed however that it is of a limited scope as the reaction products proved to be dependent on the nature of the reacting aryl hydrazones. Multicomponent reaction of $\mathbf{1 a}-\mathbf{c}$ with $\alpha, \beta$-unsaturated nitriles 2 in presence of a base has been reported to yield 3, while the reaction of $\mathbf{1 f}$ with $\mathbf{2}$ in basic medium afforded the new substituted pyrazolo[ $\left.4^{\prime}, 3^{\prime}-5,6\right]$ pyrimido[2,1-a]phthalazine-9-carbonitriles ring system 7 [12] (Scheme 1).

Microwave energy has been reported to be effective in the synthesis of small molecules in many of our previous works [13-16]. However, we noted that microwaves technology is expensive to scale up [17], in contrast to using "Q-Tube" pressure reactors, which proved to accelerate reactions of negative activation volume in a more optimal and safer manner, compared to microwaves [18]. 


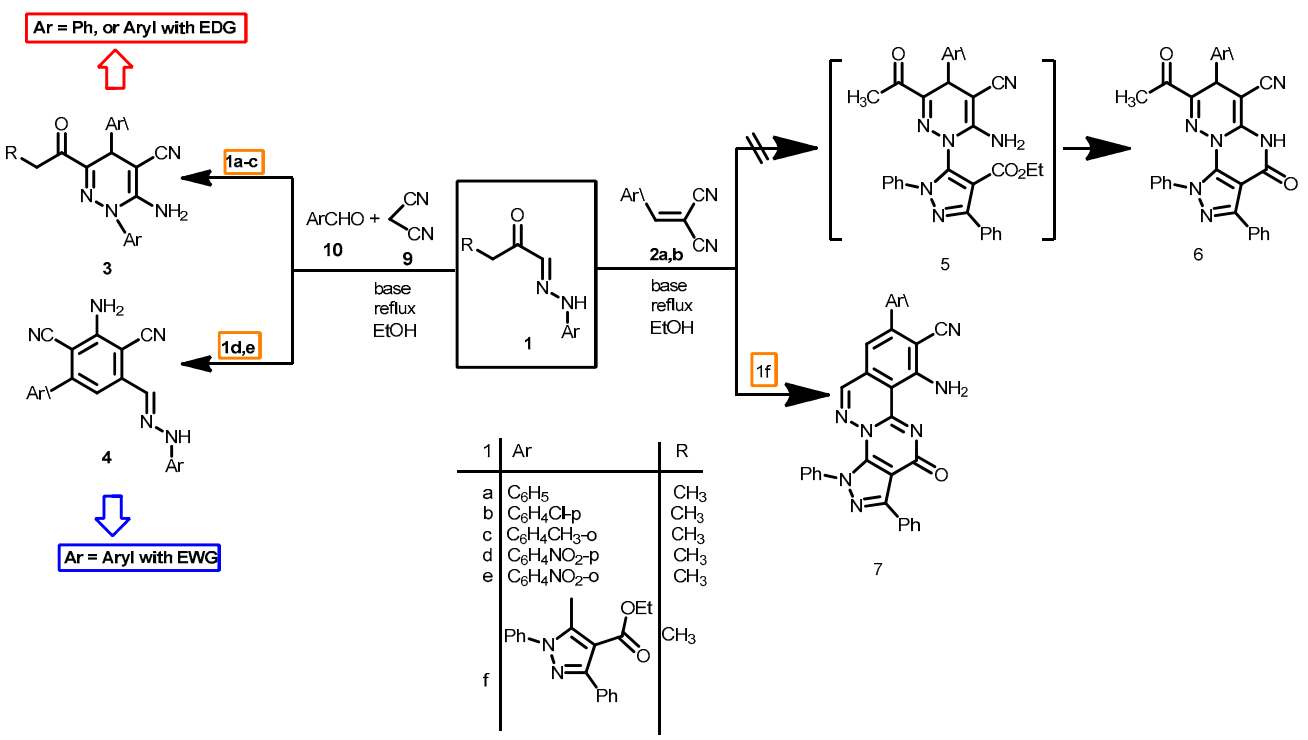

Scheme 1. The reactivity of aryl hydrazones 1 towards $\alpha, \beta$-functionally substituted cinnamonitriles.

Our research group has previously reported extensively on the use of Q-Tubes to synthesize such compounds but the reaction conditions in these published works had many limitations that altered the nature of the synthesized products $[19,20]$ (Scheme 2). The human urge to find cures for challenging diseases and improve human life leads organic chemists to be in a continuous quest to develop novel polyfunctional heterocycles, as well as developing new economical and greener technologies. Considering the promising biological activity of new compounds $\mathbf{1 2}$ where the ring system combines pyridazine and napthyridine rings, both with vast biological activities [21-24], we sought to expand this work to prove that the method proposed for the synthesis of these novel compounds is a general one by making more examples. Moreover this work has led to the proposal of a plausible reaction mechanism that would clarify and lead the way for any further work on such new ring systems.

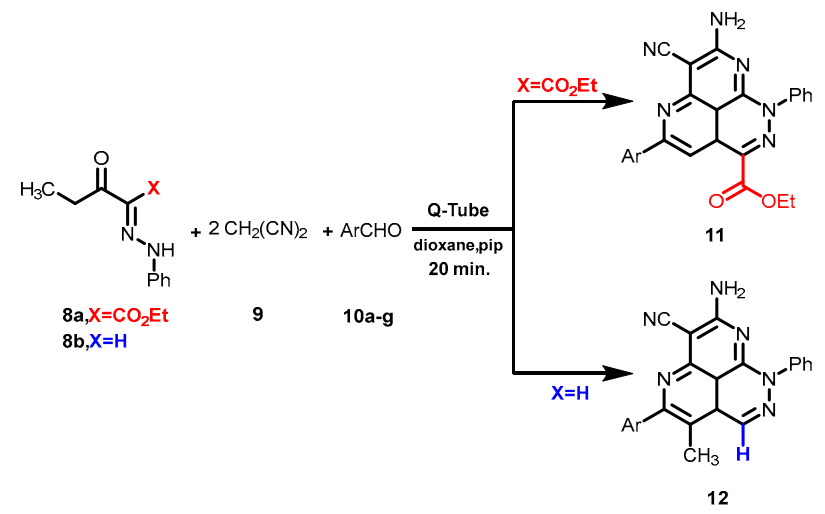

Scheme 2. Novel synthesis of the tricyclic system 11 by reacting ethyl-3-oxo-2-(2-phenylhydrazono) pentanoate (8a) with malononitrile (9) and aromatic aldehyde derivatives $\mathbf{1 0}$ in a Q-Tube.

\section{Results}

The reactions in Q-Tubes (cf. Figure 1) at $150{ }^{\circ} \mathrm{C}$ and 20 psi of 2-oxo-2-arylhydrazonals $\mathbf{1 a}, \mathbf{b}$ with aromatic aldehydes $\mathbf{1 0 a}-\mathbf{g}$ and malononitrile (9) in dioxane in the presence of piperidine afforded compounds $\mathbf{1 2 a}-\mathbf{n}$. The tricyclic systems $\mathbf{1 2}$ are formed in $72-85 \%$ yield (Scheme 3 , Table 1 ). The structure of the reaction products could be established to be pyridazino[5,4,3-de][1,6]naphthyridine 
derivatives 12a-n via spectroscopic methods (available in the supplementary materials) as well as X-ray crystal structure determination of products 12a, 12m and 12n (Figures 1-3).
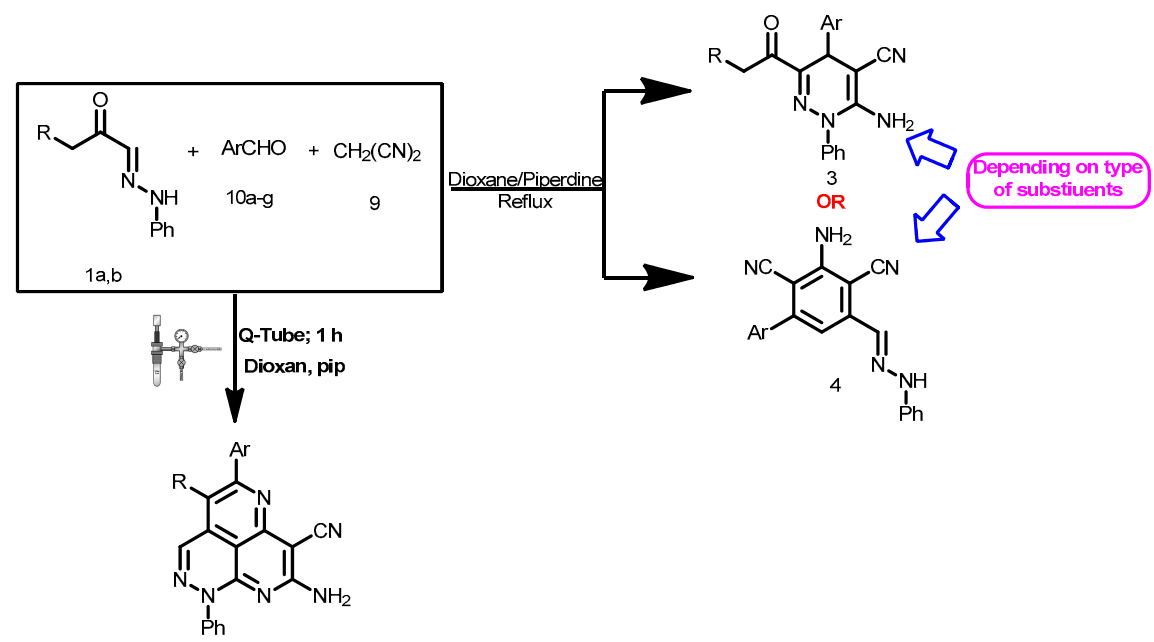

12a-n

Scheme 3. A new reaction path for the reaction of aldehyde hydrazones with malononitrile and aldehydes.

Table 1. Synthesis of pyridazino[5,4,3-de][1,6]naphthyridine derivatives 12a-n.

\begin{tabular}{ccccc}
\hline Entry & $\mathbf{R}$ & $\mathrm{Ar}$ & Yield \% & Time (min) \\
\hline $12 \mathrm{a}$ & $\mathrm{H}$ & $\mathrm{Ph}$ & 85 & 60 \\
$12 \mathrm{~b}$ & $\mathrm{H}$ & $4-\mathrm{ClC}_{6} \mathrm{H}_{4}$ & 75 & 60 \\
$12 \mathrm{c}$ & $\mathrm{H}$ & $2-\mathrm{ClC}_{6} \mathrm{H}_{4}$ & 80 & 120 \\
$12 \mathrm{~d}$ & $\mathrm{H}$ & $4-\mathrm{CH}_{3} \mathrm{C}_{6} \mathrm{H}_{4}$ & 77 & 60 \\
$12 \mathrm{e}$ & $\mathrm{H}$ & $2-\mathrm{CH}_{3} \mathrm{C}_{6} \mathrm{H}_{4}$ & 73 & 120 \\
$12 \mathrm{f}$ & $\mathrm{H}$ & $4-\mathrm{O}_{2} \mathrm{NC}_{6} \mathrm{H}_{4}$ & 82 & 60 \\
$12 \mathrm{~g}$ & $\mathrm{H}$ & $2-$ furyl & 86 & 120 \\
$12 \mathrm{~h}$ & $\mathrm{CH}_{3}$ & $\mathrm{Ph}_{1}$ & 83 & 60 \\
$12 \mathrm{j}$ & $\mathrm{CH}_{3}$ & $4-\mathrm{ClC}_{6} \mathrm{H}_{4}$ & 78 & 60 \\
$12 \mathrm{k}$ & $\mathrm{CH}_{3}$ & $4-\mathrm{CH}_{3} \mathrm{C}_{6} \mathrm{H}_{4}$ & 80 & 60 \\
$12 \mathrm{l}$ & $\mathrm{CH}_{3}$ & $2-\mathrm{CH}_{3} \mathrm{C}_{6} \mathrm{H}_{4}$ & 72 & 120 \\
$12 \mathrm{~m}$ & $\mathrm{CH}_{3}$ & $4-\mathrm{O}_{2} \mathrm{NC}_{6} \mathrm{H}_{4}$ & 82 & 60 \\
$12 \mathrm{n}$ & $\mathrm{CH}_{3}$ & $2-$ furyl & 86 & 120 \\
\hline
\end{tabular}

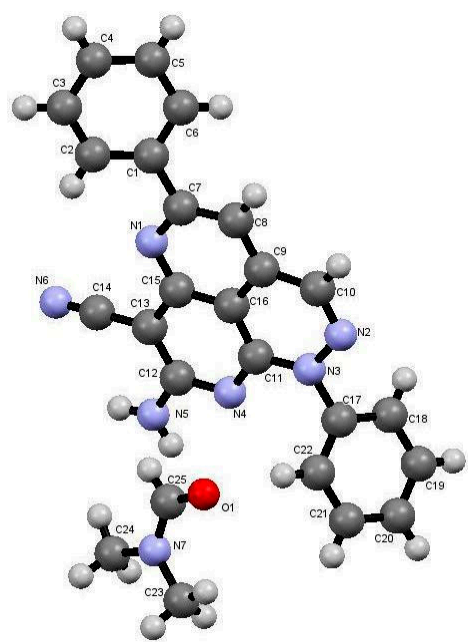

Figure 1. X-ray crystallographic structure of compound 12a [25]. 


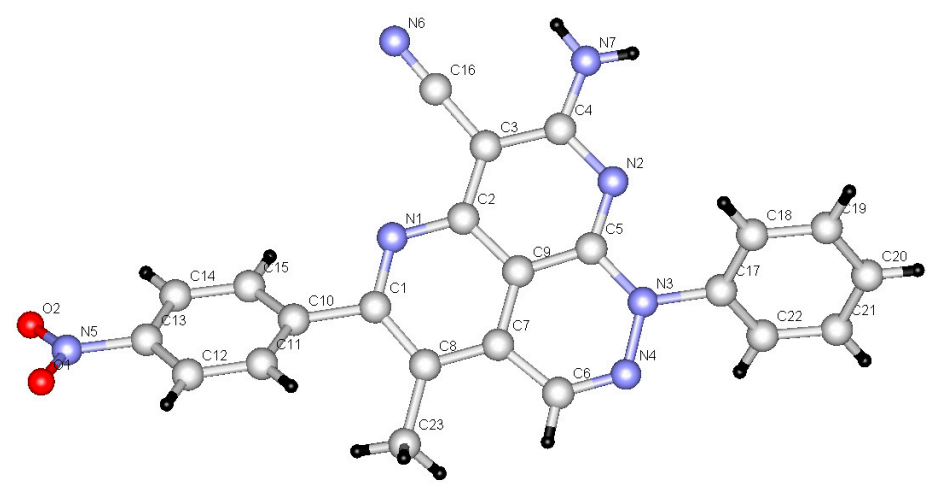

Figure 2. X-ray crystallographic structure of compound 12m [26].

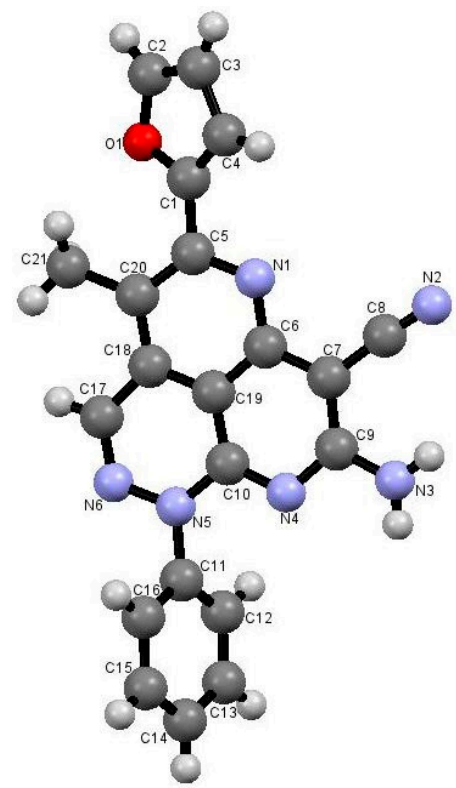

Figure 3. X-ray crystallographic structure of compound 12n [27].

\section{Discussion}

Two mechanistic pathways seem possible (Scheme 4): initial dimerization of malononitrile to yield dimer 13, that then condenses with the acyl carbonyl yielding $\mathbf{1 4}$ that cyclizes to form $\mathbf{1 5}$ (route A) [19]. This route could readily be eliminated as in our hands malononitrile could not be dimerized under the reported conditions moreover when it is considered that this dimerization it probably impossible in the absence of ethoxide or sodium hydroxide which are thought necessary for the dimerization of malononitrile [27].

Moustafa et al. also reported that the dimer $\mathbf{1 3}$ alone reacts with their arylhydrazones $\mathbf{1} \mathbf{a}, \mathbf{b}$ yielding pyridazino[5,4,3-de][1,6]naphthyridine derivatives, not condensed with pyridazine derivatives. Thus, it is almost certain that the initial step leading to formation of $\mathbf{1 2}$ is the condensation of malononitrile (9) with acyl carbonyl 18. The product $\mathbf{1 9}$ can then either cyclize into $\mathbf{2 0}$ and then $\mathbf{2 1}$ (route $\mathrm{C}$ ) or condense with an aromatic aldehyde to give 22 and then $\mathbf{2 3}$ (route D). Neither route C or D can completely be ruled out, although we believe that the aromatic aldehyde condenses initially with 19 then subsequent reactions lead to 22 that then reacts with malononitrile (9) to form 23 which cyclizes to the final product 12 (Scheme 4). 


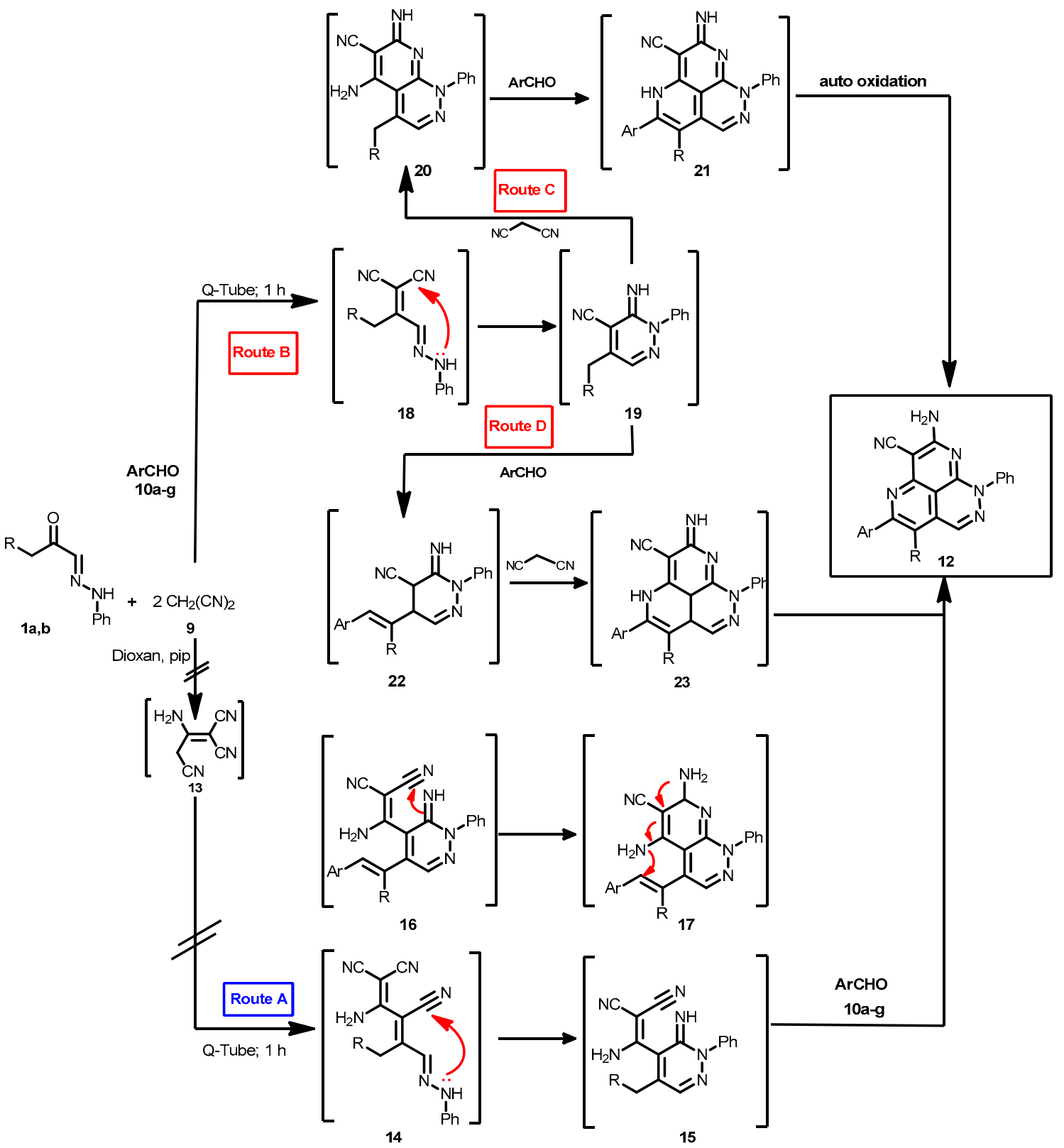

Scheme 4. The proposed mechanism for the formation of pyridazino[5,4,3-de][1,6]naphthyridine derivatives 12a-n.

\section{Materials and Methods}

\subsection{General Information}

Q-tube assisted reactions were performed in a Q-tube safe pressure reactor from Q Labtech (East Lyme, CT 06333, CT, USA, equipped with a cap/sleeve, pressure adapter (120 psi), needle adapter/needle, borosilicate glass tube, Teflon septum, and catch bottle. All reactions were monitored by using TLC with 1:1 ethyl acetate-petroleum ether as eluent and were carried out until starting materials were completely consumed. Melting points are reported uncorrected and were determined with a Sanyo (Gallenkamp, Osaka, Japan) instrument. Infrared spectra were recorded using $\mathrm{KBr}$ pellets and a FT-IR 6300 instrument (Jasco, Tokyo, Japan) and absorption bands are reported in $\mathrm{cm}^{-1}$. ${ }^{1} \mathrm{H}$ - and ${ }^{13} \mathrm{C}-\mathrm{NMR}$ spectra were determined by using a DPX instrument (Bruker, Billerica, MA, USA) at $400 \mathrm{MHz}$ or $600 \mathrm{MHz}$ for ${ }^{1} \mathrm{H}-\mathrm{NMR}$ and $100 \mathrm{MHz}$ for ${ }^{13} \mathrm{C}-\mathrm{NMR}$ and either $\mathrm{CDCl}_{3}$ or DMSO- $d_{6}$ 
solutions with TMS as internal standards. Chemical shifts are reported in ppm. Mass spectra and accurate mass measurements were made using a GCMS DFS spectrometer (Thermo, Bremen, Germany) with the EI (70 EV) mode. X-ray crystallographic structure determinations were performed by using Rapid II (Rigaku, Tokyo, Japan) and X8 Prospector (Bruker, Karlsruhe, Germany) single crystal X-ray diffractometers. All X-ray crystal structure data can be obtained free of charge from the Cambridge Crystallographic Data Centre [26-28] via www.ccdc.cam.ac.uk. The data and material are available in the Supplementary material and manuscript. Supplementary material is attached as PDF format and submitted along with the manuscript.

\subsection{General Procedures for Q-Tube-Assisted Synthesis of $\mathbf{1 2 a} \mathbf{a}-\mathbf{n}$}

2-Oxo-2-arylhydrazonals 1a,b (0.01 mol), aromatic aldehydes 13a-g (0.01 mol) and malononitrile (9) was 9 before $(0.02 \mathrm{~mol})$ in the presence of piperidine $(1 \mathrm{~mL})$ and dioxin $(20 \mathrm{~mL})$ as solvent were sequentially added in a $35 \mathrm{~mL}$ Q-tube pressure tube, furnished by Q Labtech. A Teflon septum was placed on top of the tube, and an appropriate cap was used. The mixture was heated in an oil bath at $150{ }^{\circ} \mathrm{C}$. After about $60 \mathrm{~min}$, the reaction mixture was monitored by TLC. The mixture was cooled and poured into ice-water. The solid was collected by filtration and purified by column chromatography and crystallized from ethanol.

8-Amino-1,5-diphenyl-1H-pyridazino[5,4,3-de][1,6]naphthyridine-7-carbonitrile (12a). Dark yellow crystals, Yield 85\%; m.p. 314-315 ${ }^{\circ} \mathrm{C}$; Anal. Calcd. for $\mathrm{C}_{22} \mathrm{H}_{14} \mathrm{~N}_{6}$ (362.13): C, 72.92; H, 3.89; N, 23.19. Found: C, 72.83; H, 3.79; N, 23.25. EI-HRMS: $m / z=362.1274\left(\mathrm{MH}^{+}\right) ; \mathrm{C}_{22} \mathrm{H}_{14} \mathrm{~N}_{6}$ requires: $m / z=362.1279\left(\mathrm{MH}^{+}\right)$; ${ }^{1} \mathrm{H}-\mathrm{NMR}\left(400 \mathrm{MHz}, \mathrm{DMSO}-d_{6}\right): \delta=7.06$ (br, 2H, NH${ }_{2}, \mathrm{D}_{2} \mathrm{O}$ exchangeable), 7.44-7.66 (m, H, Ph-H, CH), 8.19-8.22 (m, 2H, Ph-H), 8.38 (s, 1H, CH); ${ }^{13} \mathrm{C}-\mathrm{NMR}\left(100 \mathrm{MHz}, \mathrm{DMSO}-d_{6}\right): \delta=162.0,161.2,154.5,150.9$, 141.7, 138.7, 137.8, 133.2, 130.3, 128.8 (2C), 128.7 (2C), 128.1, 127.1 (2C), 126.3 (2C), 116.8, 108.8, 104.6, 73.3. MS: $m / z(\%) 362.2\left(\mathrm{M}^{+}, 100\right), 334(10), 181$ (10), 77 (5).

8-Amino-5-(4-chlorophenyl)-1-phenyl-1H-pyridazino[5,4,3-de][1,6]naphthyridine-7-carbonitrile (12b). Green crystals, yield 75\%; m.p. 340-341 ${ }^{\circ} \mathrm{C}$; Anal. Calcd. for $\mathrm{C}_{22} \mathrm{H}_{13} \mathrm{ClN}_{6}$ (396.06): C, 66.59; H, 3.30; N, 21.18. Found: C, 66.70; H, 3.35; N, 21.20. EI-HRMS: $m / z=396.0885\left(\mathrm{MH}^{+}\right) ; \mathrm{C}_{22} \mathrm{H}_{13} \mathrm{~N}_{6}{ }^{35} \mathrm{Cl}$ requires: $m / z$ $396.0890\left(\mathrm{MH}^{+}\right) ;{ }^{1} \mathrm{H}-\mathrm{NMR}\left(400 \mathrm{MHz}, \mathrm{DMSO}-d_{6}\right): \delta=7.10\left(\mathrm{br}, 2 \mathrm{H}, \mathrm{NH}_{2}, \mathrm{D}_{2} \mathrm{O}\right.$ exchangeable), 7.45-7.66 (m, 8H, Ph-H, CH), 8.22-8.24 (m, 2H, Ph-CH), 8.38 (s, 1H, CH); ${ }^{13} \mathrm{C}-\mathrm{NMR}\left(100 \mathrm{MHz}, \mathrm{DMSO}-d_{6}\right)$ : $\delta=162.1,160.0,154.6,151.0,141.7,138.7,136.7,133.4,130.3,129.1(2 \mathrm{C}), 128.9(2 \mathrm{C}), 128.2,126.6(2 \mathrm{C})$, 124.6 (2C), 118.0, 109.1, 104.6, 56.0. MS: $m / z$ (\%) $396.1\left(\mathrm{M}^{+}, 100\right), 368(10), 198$ (10), 166 (5), 77 (5).

8-Amino-5-(3-chlorophenyl)-1-phenyl-1H-pyridazino[5,4,3-de][1,6]naphthyridine-7-carbonitrile (12c). Green crystals, yield 80\%; m.p. 314-316 ${ }^{\circ} \mathrm{C}$; Anal. Calcd. for $\mathrm{C}_{22} \mathrm{H}_{13} \mathrm{ClN}_{6}$ (396.06): C, 66.59; $\mathrm{H}, 3.30 ; \mathrm{N}$, 21.18. Found: $\mathrm{C}, 66.57 ; \mathrm{H}, 3.33 ; \mathrm{N}, 21.12$. EI-HRMS: $m / z=396.0885\left(\mathrm{MH}^{+}\right) ; \mathrm{C}_{22} \mathrm{H}_{13} \mathrm{~N}_{6}{ }^{35} \mathrm{Cl}$ requires: $m / z=396.0890\left(\mathrm{MH}^{+}\right) ;{ }^{1} \mathrm{H}-\mathrm{NMR}\left(400 \mathrm{MHz}, \mathrm{DMSO}-d_{6}\right): \delta=7.11\left(\mathrm{br}, 2 \mathrm{H}, \mathrm{NH}_{2}, \mathrm{D}_{2} \mathrm{O}\right.$ exchangeable), $7.27(\mathrm{~s}, 1 \mathrm{H}, \mathrm{CH}), 7.45-7.67(\mathrm{~m}, 9 \mathrm{H}, \mathrm{Ph}-\mathrm{H}), 8.45(\mathrm{~s}, 1 \mathrm{H}, \mathrm{CH}) ;{ }^{13} \mathrm{C}-\mathrm{NMR}\left(100 \mathrm{MHz}, \mathrm{DMSO}-d_{6}\right): \delta=162.2$, 162.0, 154.6, 151.1, 141.7, 138.8, 138.5, 132.4, 131.4, 131.0, 130.6, 129.9 (2C), 128.8, 128.2, 127.4, $126.4(2 \mathrm{C})$, 116.8, 108.9, 108.8, 73.1. MS: $m / z$ (\%) $396.1\left(\mathrm{M}^{+}, 100\right), 361$ (15), 334 (5), 198 (10), 166 (5), 77 (5).

8-Amino-1-phenyl-5-p-tolyl-1H-pyridazino[5,4,3-de][1,6]naphthyridine-7-carbonitrile (12d). Faint green crystals, yield 77\%; m.p. 340-341 ${ }^{\circ} \mathrm{C}$; Anal. Calcd. for $\mathrm{C}_{23} \mathrm{H}_{16} \mathrm{~N}_{6}$ (376.14): C, 73.39; $\mathrm{H}, 4.28 ; \mathrm{N}$, 22.33. Found: C, 73.34; $\mathrm{H}, 4.30 ; \mathrm{N}, 22.28$. EI-HRMS: $m / z=376.1430\left(\mathrm{MH}^{+}\right) ; \mathrm{C}_{23} \mathrm{H}_{16} \mathrm{~N}_{6}$ requires: $m / z=376.1436\left(\mathrm{MH}^{+}\right) ;{ }^{1} \mathrm{H}-\mathrm{NMR}\left(400 \mathrm{MHz}, \mathrm{DMSO}-d_{6}\right): \delta=2.39,2.51\left(\mathrm{~s}, 3 \mathrm{H}, \mathrm{CH}_{3}\right), 7.05\left(\mathrm{br}, 2 \mathrm{H}, \mathrm{NH}_{2}\right.$, $\mathrm{D}_{2} \mathrm{O}$ exchangeable), 7.34-7.65 (m, 8H, Ph-H, CH), 8.09-8.11 (m, 2H, Ph-H), $8.36(\mathrm{~s}, 1 \mathrm{H}, \mathrm{CH}) ;{ }^{13} \mathrm{C}-\mathrm{NMR}$ $\left(100 \mathrm{MHz}, \mathrm{DMSO}-d_{6}\right): \delta=162.2,161.2,154.4,150.9,141.7,140.2,138.8,135.1,133.2,129.4(2 \mathrm{C}), 128.8(2 \mathrm{C})$, $128.1,127.1$ (2C), 126.3 (2C), 116.9, 108.7, 104.2, 73.3, 20.9. MS: $m / z$ (\%) $376.2\left(\mathrm{M}^{+}, 100\right), 348$ (10), 188 (10), 77 (5).

8-Amino-1-phenyl-5-m-tolyl-1H-pyridazino[5,4,3-de][1,6]naphthyridine-7-carbonitrile (12e). Green crystals, yield 73\%; m.p. 280-281 ${ }^{\circ} \mathrm{C}$; Anal. Calcd. for $\mathrm{C}_{23} \mathrm{H}_{16} \mathrm{~N}_{6}$ (376.14): C, 73.39; H, 4.28; N, 22.33. Found: C, 
73.38; $\mathrm{H}, 4.27 ; \mathrm{N}, 22.31$. EI-HRMS: $m / z=376.1431\left(\mathrm{MH}^{+}\right) ; \mathrm{C}_{23} \mathrm{H}_{16} \mathrm{~N}_{6}$ requires: $m / z=376.1436\left(\mathrm{MH}^{+}\right)$; ${ }^{1} \mathrm{H}-\mathrm{NMR}\left(400 \mathrm{MHz}, \mathrm{DMSO}-d_{6}\right): \delta=2.43,2.51\left(\mathrm{~s}, 3 \mathrm{H}, \mathrm{CH}_{3}\right), 7.07\left(\mathrm{br}, 2 \mathrm{H}, \mathrm{NH}_{2}, \mathrm{D}_{2} \mathrm{O}\right.$ exchangeable), $7.18(\mathrm{~s}, 1 \mathrm{H}, \mathrm{CH}), 7.34-7.66(\mathrm{~m}, 9 \mathrm{H}, \mathrm{Ph}-\mathrm{H}), 8.40(\mathrm{~s}, 1 \mathrm{H}, \mathrm{CH}) ;{ }^{13} \mathrm{C}-\mathrm{NMR}\left(100 \mathrm{MHz}, \mathrm{DMSO}-d_{6}\right): \delta=165.2$, 162.0, 154.3, 151.1, 141.7, 139.6, 138.7, 135.8, 133.7, 130.8, 129.3, 128.9, 128.2 (2C), 128.1, 126.3 (2C), 125.9, 116.9, 108.3, 108.2, 73.3, 20.3. MS: $m / z(\%) 376\left(\mathrm{M}^{+}, 50\right), 375$ (100), 348 (10), 255 (10), 187 (10), 77 (5).

8-Amino-5-(4-nitrophenyl)-1-phenyl-1H-pyridazino[5,4,3-de][1,6]naphthyridine-7-carbonitrile (12f). Dark brown crystals, yield 82\%; m.p. 397-398 ${ }^{\circ} \mathrm{C}$; Anal. Calcd. for $\mathrm{C}_{22} \mathrm{H}_{13} \mathrm{~N}_{7} \mathrm{O}_{2}$ (407.11): C, 64.86; $\mathrm{H}, 3.22$; $\mathrm{N}, 24.07$. Found: $\mathrm{C}, 64.75 ; \mathrm{H}, 3.10 ; \mathrm{N}, 24.12$. EI-HRMS: $m / z=407.1125\left(\mathrm{MH}^{+}\right) ; \mathrm{C}_{22} \mathrm{H}_{13} \mathrm{O}_{2} \mathrm{~N}_{7}$ requires: $m / z=407.1131\left(\mathrm{MH}^{+}\right) ;{ }^{1} \mathrm{H}-\mathrm{NMR}\left(400 \mathrm{MHz}, \mathrm{DMSO}-d_{6}\right): \delta=7.18\left(\mathrm{br}, 2 \mathrm{H}, \mathrm{NH}_{2}, \mathrm{D}_{2} \mathrm{O}\right.$ exchangeable), 7.48-7.68 (m, 5H, Ph-H, CH), 7.93, 8.244 (d, 1H, CH-pyridazine), 8.40-8.48 (m, 4H, Ph-H, CH), 9.20, 9.41 (s, 1H, CH). MS: $m / z(\%) 407.2\left(\mathrm{M}^{+}, 100\right), 361$ (20), 334 (10), 180 (10), 77 (5).

8-Amino-5-(furan-2-yl)-1-phenyl-1H-pyridazino[5,4,3-de][1,6]naphthyridine-7-carbonitrile (12g). Dark green crystals, yield 86\%; m.p. $345-346{ }^{\circ} \mathrm{C}$; Anal. Calcd. for $\mathrm{C}_{20} \mathrm{H}_{12} \mathrm{~N}_{6} \mathrm{O}$ (352.11): C, 68.18; H, 3.43; N, 23.85. Found: $\mathrm{C}, 68.21 ; \mathrm{H}, 3.52 ; \mathrm{N}, 23.77$. EI-HRMS: $m / z=352.1066\left(\mathrm{MH}^{+}\right) ; \mathrm{C}_{20} \mathrm{H}_{12} \mathrm{O}_{1} \mathrm{~N}_{6}$ requires: $m / z=352.1072\left(\mathrm{MH}^{+}\right) ;{ }^{1} \mathrm{H}-\mathrm{NMR}\left(400 \mathrm{MHz}, \mathrm{DMSO}-d_{6}\right): \delta=6.74-6.75(\mathrm{~m}, 1 \mathrm{H}$, furyl-H) $7.05(\mathrm{br}, 2 \mathrm{H}$, $\mathrm{NH}_{2}, \mathrm{D}_{2} \mathrm{O}$ exchangeable), 7.24-7.97 (m, 8H, Ph-H, furyl-H, CH), $8.42(\mathrm{~s}, 1 \mathrm{H}, \mathrm{CH}) ;{ }^{13} \mathrm{C}-\mathrm{NMR}(100 \mathrm{MHz}$, DMSO- $\left.d_{6}\right): \delta=162.0,154.6,153.2,152.7,150.8,145.6,141.7,138.6,133.2,128.8(2 \mathrm{C}), 128.2,126.3(2 \mathrm{C})$, 116.8, 112.7, 11.8, 108.6, 102.7, 73.0. MS: $m / z(\%) 352.1\left(\mathrm{M}^{+}, 100\right), 324$ (5), 176 (10), 77 (5).

8-Amino-4-methyl-1,5-diphenyl-1H-pyridazino[5,4,3-de][1,6]naphthyridine-7-carbonitrile (12h). Yellow crystals, yield 83\%; m.p. $364-365{ }^{\circ} \mathrm{C}$; Anal. Calcd. for $\mathrm{C}_{23} \mathrm{H}_{16} \mathrm{~N}_{6}$ (376.14): C, 73.39; $\mathrm{H}, 4.28$; N, 22.33. Found: $\mathrm{C}, 73.35 ; \mathrm{H}, 4.15 ; \mathrm{N}, 22.41$. EI-HRMS: $m / z=376.1431\left(\mathrm{MH}^{+}\right) ; \mathrm{C}_{23} \mathrm{H}_{16} \mathrm{~N}_{6}$ requires: $m / z=376.1436\left(\mathrm{MH}^{+}\right) ;{ }^{1} \mathrm{H}-\mathrm{NMR}\left(400 \mathrm{MHz}, \mathrm{DMSO}-d_{6}\right): \delta=2.34,2.50\left(\mathrm{~s}, 3 \mathrm{H}, \mathrm{CH}_{3}\right), 6.95\left(\mathrm{br}, 2 \mathrm{H}, \mathrm{NH}_{2}\right.$, $\mathrm{D}_{2} \mathrm{O}$ exchangeable), 7.45-7.66 (m, $\left.10 \mathrm{H}, \mathrm{Ph}-\mathrm{H}, \mathrm{CH}\right), 8.56(\mathrm{~s}, 1 \mathrm{H}, \mathrm{CH}) ;{ }^{13} \mathrm{C}-\mathrm{NMR}\left(100 \mathrm{MHz}, \mathrm{DMSO}-d_{6}\right)$ : $\delta=164.5,161.6,152.3,150.6,141.8,140.0,136.9,131.0,129.0(2 \mathrm{C}), 128.8(2 \mathrm{C}), 128.5,128.2,128.0(2 \mathrm{C})$, $126.3(2 \mathrm{C}), 117.1,114.1,108.9,72.6,13.9 . \mathrm{MS}: m / z(\%) 376.2\left(\mathrm{M}^{+}, 100\right), 368$ (10), $348(5), 255$ (5), $188(10)$, 97 (10), 57 (5).

8-Amino-5-(4-chlorophenyl)-4-methyl-1-phenyl-1H-pyridazino[5,4,3-de][1,6]naphthyridine-7-carbonitrile (12j). Dark yellow crystals, yield 78\%; m.p. $345-346^{\circ} \mathrm{C}$; Anal. Calcd. for $\mathrm{C}_{23} \mathrm{H}_{15} \mathrm{ClN}_{6}$ (410.1): C, 67.24; $\mathrm{H}$, 3.68; $\mathrm{N}, 20.45$. Found: $\mathrm{C}, 67.27 ; \mathrm{H}, 3.56 ; \mathrm{N}, 20.45$. EI-HRMS: $m / z=410.1041\left(\mathrm{MH}^{+}\right) ; \mathrm{C}_{23} \mathrm{H}_{15} \mathrm{~N}_{6}{ }^{35} \mathrm{Cl}$ requires: $m / z=410.1047\left(\mathrm{MH}^{+}\right) ;{ }^{1} \mathrm{H}-\mathrm{NMR}\left(400 \mathrm{MHz}, \mathrm{DMSO}-d_{6}\right): \delta=2.35,2.50\left(\mathrm{~s}, 3 \mathrm{H}, \mathrm{CH}_{3}\right), 6.90(\mathrm{br}$, $2 \mathrm{H}, \mathrm{NH}_{2}, \mathrm{D}_{2} \mathrm{O}$ exchangeable), 7.45-7.66 (m, 9H, Ph- $\left.\mathrm{H}, \mathrm{CH}\right), 8.58(\mathrm{~s}, 1 \mathrm{H}, \mathrm{CH}) ;{ }^{13} \mathrm{C}-\mathrm{NMR}(100 \mathrm{MHz}$, DMSO- $\left.d_{6}\right): \delta=163.8,162.1,156.4,151.1,142.3,139.4,137.3,134.0,132.5,131.7,131.3(2 \mathrm{C}), 129.3(2 \mathrm{C})$, 128.6 (2C), 126.7 (2C), 117.2, 114.8, 109.7, 73.5, 14.3. MS: $m / z(\%) 410.1\left(\mathrm{M}^{+}, 100\right), 374(10), 346(5)$, 255 (5), 205 (5), 187 (10), 173 (5), 97 (5), 77 (5).

8-Amino-4-methyl-1-phenyl-5-p-tolyl-1H-pyridazino[5,4,3-de][1,6]naphthyridine-7-carbonitrile (12k). Dark orange crystals, yield $80 \%$; m.p. $370-371{ }^{\circ} \mathrm{C}$; Anal. Calcd. for $\mathrm{C}_{24} \mathrm{H}_{18} \mathrm{~N}_{6}$ (390.16): C, 73.83; H, 4.65; $\mathrm{N}, 21.52$. Found: $\mathrm{C}, 73.88 ; \mathrm{H}, 4.59 ; \mathrm{N}, 21.60$. EI-HRMS: $m / z=390.1587\left(\mathrm{MH}^{+}\right) ; \mathrm{C}_{24} \mathrm{H}_{18} \mathrm{~N}_{6}$ requires: $m / z=390.1593\left(\mathrm{MH}^{+}\right) ;{ }^{1} \mathrm{H}-\mathrm{NMR}\left(400 \mathrm{MHz}, \mathrm{DMSO}-d_{6}\right): \delta=2.36\left(\mathrm{~s}, 3 \mathrm{H}, \mathrm{CH}_{3}\right), 2.41\left(\mathrm{~s}, 3 \mathrm{H}, \mathrm{CH}_{3}\right), 6.93(\mathrm{br}$, $2 \mathrm{H}, \mathrm{NH}_{2}, \mathrm{D}_{2} \mathrm{O}$ exchangeable), 7.34-7.67 (m, 9H, Ph-H, CH), $8.56(\mathrm{~s}, 1 \mathrm{H}, \mathrm{CH}) ;{ }^{13} \mathrm{C}-\mathrm{NMR}(100 \mathrm{MHz}$, DMSO- $\left.d_{6}\right): \delta=163.9,161.6,152.4,150.7,141.8,140.2,137.2,129.0(2 \mathrm{C}), 128.8(2 \mathrm{C}), 128.6(2 \mathrm{C}), 127.1$, 126.3 (2C), 129.1, 124.5, 119.1, 114.8, 109.7, 72.6, 20.8, 14.0. MS: $m / z(\%) 390.2\left(\mathrm{M}^{+}, 100\right), 375$ (5), 269 (5), 187 (5), 77 (5).

8-Amino-4-methyl-1-phenyl-5-m-tolyl-1H-pyridazino[5,4,3-de][1,6]naphthyridine-7-carbonitrile (121). Yellow crystals, yield 72\%; m.p. $284-285^{\circ} \mathrm{C}$; Anal. Calcd. for $\mathrm{C}_{24} \mathrm{H}_{18} \mathrm{~N}_{6}$ (390.16): C, 73.83; H, 4.65; N, 21.52. Found: $\mathrm{C}, 73.81 ; \mathrm{H}, 4.68 ; \mathrm{N}, 21.55$. EI-HRMS: $m / z=390.1587\left(\mathrm{MH}^{+}\right) ; \mathrm{C}_{24} \mathrm{H}_{18} \mathrm{~N}_{6}$ requires: $m / z=390.1592$ $\left(\mathrm{MH}^{+}\right)$; IR: 3489, $3336\left(\mathrm{NH}_{2}\right), 2200(\mathrm{CN}) ;{ }^{1} \mathrm{H}-\mathrm{NMR}\left(400 \mathrm{MHz}, \mathrm{DMSO}-d_{6}\right): \delta=2.08\left(\mathrm{~s}, 3 \mathrm{H}, \mathrm{CH}_{3}\right), 2.11$ (s, 3H, $\left.\mathrm{CH}_{3}\right), 6.96$ (br, 2H, NH${ }_{2}, \mathrm{D}_{2} \mathrm{O}$ exchangeable), 7.19-7.66 (m, 9H, Ph-H, CH), $8.53(\mathrm{~s}, 1 \mathrm{H}, \mathrm{CH})$; ${ }^{13} \mathrm{C}-\mathrm{NMR}\left(100 \mathrm{MHz}, \mathrm{DMSO}-d_{6}\right): \delta=165.5,161.5,152.4,150.7,141.8,139.8,136.9,134.8,130.6,130.0$, 
128.9 (2C), 128.2 (2C), 126.3 (2C), 125.6 (2C), 117.1, 114.7, 109.0, 72.6, 19.0, 13.0. MS: $\mathrm{m} / z(\%) 390.2\left(\mathrm{M}^{+}\right.$, 50), 375 (100), 346 (5), 255 (5), 195 (5), 187 (15), 173 (10), 129 (5), 77 (5).

8-Amino-4-methyl-5-(4-nitrophenyl)-1-phenyl-1H-pyridazino[5,4,3-de][1,6]naphthyridine-7-carbonitrile (12m). Dark yellow crystals, yield 82\%; m.p. 368-369 ${ }^{\circ} \mathrm{C}$; Anal. Calcd. for $\mathrm{C}_{23} \mathrm{H}_{15} \mathrm{~N}_{7} \mathrm{O}_{2}$ (421.13): C, 65.55; $\mathrm{H}, 3.59 ; \mathrm{N}, 23.27$. Found: $\mathrm{C}, 65.59 ; \mathrm{H}, 3.63 ; \mathrm{N}, 23.31$. EI-HRMS: $m / z=421.1282\left(\mathrm{MH}^{+}\right) ; \mathrm{C}_{23} \mathrm{H}_{15} \mathrm{O}_{2} \mathrm{~N}_{7}$ requires: $m / z=421.1287\left(\mathrm{MH}^{+}\right) ;{ }^{1} \mathrm{H}-\mathrm{NMR}\left(400 \mathrm{MHz}, \mathrm{DMSO}-d_{6}\right): \delta=2.37,2.53\left(\mathrm{~s}, 3 \mathrm{H}, \mathrm{CH}_{3}\right), 6.82(\mathrm{br}$, $2 \mathrm{H}, \mathrm{NH}_{2}, \mathrm{D}_{2} \mathrm{O}$ exchangeable), 7.47-8.38 (m, 9H, Ph-H, CH), 8.58, 8.58 (s, 1H, CH); ${ }^{13} \mathrm{C}-\mathrm{NMR}(100 \mathrm{MHz}$, DMSO-d $\left.d_{6}\right): \delta=14.56,49.5,105,124.12(2 \mathrm{C}), 127.10(2 \mathrm{C}), 129.08,129.70(2 \mathrm{C}), 131.31(4 \mathrm{C}), 135.61,137.67$, 142.69, 162.56, 163.25; MS: m/z (\%) $421.2\left(\mathrm{M}^{+}, 100\right), 390$ (15), 374 (25), 348 (10), 255 (5), 187 (10), 77 (5).

8-Amino-5-(furan-2-yl)-4-methyl-1-phenyl-1H-pyridazino[5,4,3-de][1,6]naphthyridine-7-carbonitrile (12n). Dark green crystals, yield 86\%; m.p. 368-369 ${ }^{\circ} \mathrm{C}$; Anal. Calcd. for $\mathrm{C}_{21} \mathrm{H}_{14} \mathrm{~N}_{6} \mathrm{O}$ (366.12): C, 68.84; $\mathrm{H}$, 3.85; N, 22.94. Found: C, 68.89; H, 3.78; N, 22.88. EI-HRMS: $m / z=366.1223\left(\mathrm{MH}^{+}\right) ; \mathrm{C}_{21} \mathrm{H}_{14} \mathrm{O}_{1} \mathrm{~N}_{6}$ requires: $m / z=366.1229\left(\mathrm{MH}^{+}\right) ;{ }^{1} \mathrm{H}-\mathrm{NMR}\left(400 \mathrm{MHz}, \mathrm{DMSO}-d_{6}\right): \delta=2.50\left(\mathrm{~s}, 3 \mathrm{H}, \mathrm{CH}_{3}\right), 6.72-6.73(\mathrm{~m}$, $1 \mathrm{H}$, furyl-H), 6.91 (br, 2H, NH $\mathrm{N}_{2}, \mathrm{D}_{2} \mathrm{O}$ exchangeable), 7.19-7.97 (m, 7H, Ph-H, furyl-H), 8.52 (s, 1H, CH); ${ }^{13} \mathrm{C}-\mathrm{NMR}\left(100 \mathrm{MHz}, \mathrm{DMSO}-d_{6}\right): \delta=161.6,153.0,152.4,152.2,150.4,145.1,141.8,136.8,131.8,128.9(2 \mathrm{C})$, $128.2,126.3$ (2C), 117.0, 114.3, 113.2, 112.0, 108.8, 72.3, 13.2. MS: $m / z(\%) 366.1\left(\mathrm{M}^{+}, 100\right), 337$ (5), 311 (5), 183 (5), 77 (10).

\section{Conclusions}

Synthesis of 2-amino-1,4-dihydropyridazines by reacting arylhydrazonals, active methylene nitriles and aromatic aldehydes has been found of very limited scope. We show here that under pressure the sequence of this multicomponent reaction changes as the initial step with the least activation volume predominates, and in this way a novel route to pyridazino[5,4,3-de][1,6]naphthyridines could be developed. Our observations open the route for discovering new multicomponent reactions under pressure, thus it is recommended to expand this technique.

Supplementary Materials: Supplementary Materials are available online.

Acknowledgments: This project was funded by the Deanship of Scientific Research (DSR), King Abdulaziz University, Jeddah, under grant No. (2-363-36-RG). The authors, therefore, would like to express their deep gratitude to the DSR technical and financial support.

Author Contributions: The main part of the work was carried out by Majdah A. AL-Johani, Sameera M. Mousally and Noha Hilmy Elnagdi, under the direct supervision of Khadijah M. Al-Zaydi and Norah F. Alqahtani. Conceptually the work was designed by Mohamed H. Elnagdi, Khadijah M. Al-Zaydi and Norah F. Alqahtani. All authors read and approved the final manuscript.

Conflicts of Interest: The authors declare that they have no competing interests.

\section{References}

1. Asif, M. Some Recent Approaches of Biologically Active Substituted Pyridazine and Phthalazine Drugs. Curr. Med. Chem. 2012, 19, 2984-2991. [CrossRef] [PubMed]

2. Singh, A.K.; Hegde, G.L.; Khanum, S.A.; Shashikanth, S. Synthesis and Pharmacological Activity of 4-Aryl-Thieno-(2,3-d)-Pyridazines. Indian J. Pharm. Sci. 2005, 67, 210-215.

3. Tucaliuc, R.A.; Cotea, V.V.; Niculaua, M.; Tuchilus, C.; Mantu, D.; Mangalagiu, I.I. New pyridazine-fluorine derivatives: Synthesis, chemistry and biological activity. Eur. J. Med. Chem. 2013, 67, 367-372. [CrossRef] [PubMed]

4. Gao, Q.; Zhu, Y.; Lian, M.; Liu, M.; Yuan, J.; Yin, G.; Wu, A. Unexpected C-C Bond Cleavage: A Route to 3, 6-Diarylpyridazines and 6-Arylpyridazin-3-ones from 1,3-Dicarbonyl Compounds and Methyl Ketones. J. Org. Chem. 2012, 77, 9865-9870. [CrossRef] [PubMed]

5. Kessler, S.N.; Wegner, H.A. One-Pot Synthesis of Phthalazines and Pyridazino-aromatics: A Novel Strategy for Substituted Naphthalenes. Org. Lett. 2012, 14, 3268-3271. [CrossRef] [PubMed] 
6. Poschenrieder, H.; Stachel, H.-D. Synthesis of pyrrolo[3,4-c]pyridazines. J. Heterocycl. Chem. 1995, 32, 1457-1460. [CrossRef]

7. Behbehani, H.; Ibrahim, H.M. Microwave-Assisted Synthesis in Water: First One-Pot Synthesis of A Novel Class of Polysubstituted benzo[4,5]imidazo[1,2-b]pyridazines via Intramolecular SNAr. RSC Adv. 2015, 5, 89226-89237. [CrossRef]

8. Elnagdi, M.H.; Moustafa, M.S.; Sadek, K.U. Green Synthesis of Biologically Relevant Azoles and Azines Derivatives, 1st ed.; Lap Lambert Acadimic Publishing: Saarbrücken, Germany, 2014; p. 64. ISBN 9783659551086.

9. Ghozlan, S.A.S.; Abdelhamid, I.A.; Hassaneen, H.M.; Elnagdi, M.H. Studies with Enamines and Azaenamines: A novel Efficient Route to 6-amino-1,4-dihydropyridazines and their Condensed Derivatives. J. Heterocycl. Chem. 2007, 44, 105-108. [CrossRef]

10. Al-Mousawi, S.M.; Moustafa, M.S.; Abdelhamid, I.A.; Elnagdi, M.H. Reassignment of the Structures of Condensation Products of $\alpha$-keto $\alpha$-formylarylhydrazones with Ethyl Cyanoacetate: A Novel Route to ethyl 5-arylazo-2-hydroxynicotinates. Tetrahedron Lett. 2011, 52, 202-204. [CrossRef]

11. Ackermann, L.; Gunnoe, T.B.; Habgood, L.G. Catalytic Hydroarylation of Carbon-Carbon Multiple Bonds; Wiley-VCH Verlag Gmbh \& Co. KGaA: Weinheim, Germany, 2017; pp. 51-58. ISBN 9783527340132.

12. Ghozlan, S.A.S.; Abdelmoniem, A.M.; Butenschon, H.; Abdelhamid, I.A. Discrepancies in The Reactivity Pattern of Azaenamines towards Cinnamonitriles: Synthesis of Novel aza-steroid Analogues. Tetrahedron 2015, 71, 1413-1418. [CrossRef]

13. Al-Zaydi, K.M. Microwave Assisted Synthesis, Part 1: Rapid Solventless Synthesis of 3-Substituted Coumarins and Benzocoumarins by Microwave Irradiation of the Corresponding Enaminones. Molecules 2003, 8, 541-555. [CrossRef]

14. Al-Zaydi, K.M.; Borik, R.M.; Elnagdi, M.H. Arylhydrazononitriles as Precursors to 2-Substituted 1,2,3-triazoles and 4-amino-5-cyano-pyrazole Derivatives Utilizing Microwave and Ultrasound Irradiation. Green Chem. Lett. Rev. 2012, 5, 241-250. [CrossRef]

15. Al-Zaydi, K.M.; Nhari, L.M.; Borik, R.M.; Elnagdi, M.H. Green Technologies in Organic Synthesis: Self-Condensation of Enamines, Enaminones and EnaminoestersUnder Microwave Irradiation in Ionic Liquid. Green Chem. Lett. Rev. 2010, 3, 93-99. [CrossRef]

16. Al-Zaydi, K.M.; Borik, R.M.; Elnagdi, M.H. 2-Arylhydrazonopropanals as Building Blocks in Heterocyclic Chemistry: Microwave Assisted Condensation of 2-Aryl-hydrazonopropanals with Amines and Active Methylene Reagents. Molecules 2003, 8, 910-923. [CrossRef]

17. Kappe, C.O.; Stadler, A. Microwaves in Organic and Medicinal Chemistry; John Wiley \& Sons: Hoboken, NJ, USA, 2005; pp. 3-5. ISBN 9783527606559. [CrossRef]

18. Kappe, C.O.; Dallinger, D.; Murphree, S.S. Practical Microwave Synthesis for Organic Chemists-Strategies; John Wiley \& Sons: Hoboken, NJ, USA, 2009; pp. 2-4. ISBN 9783527623907. [CrossRef]

19. Moustafa, M.S.; Al-Mousawi, S.M.; Abdelhamid, I.A.; Elnagdi, M.H. Use of A Novel Multicomponent Reaction Under High Pressure for The Efficient Construction of A New Pyridazino[5,4,3-de][1,6] naphthyridine Tricyclic System. RSC Adv. 2016, 93, 90840-90845. [CrossRef]

20. Sadek, K.U.; Selim, M.A.; .Alnajjar, A.; Atallah, M.; Elnagdi, M.H. Multicomponent Reactions under Increased Pressure: On the Reaction of Arylhydrazonals, Aromatic Aldehydes and Malononitrile in Q-Tube. Chem. Eur. J. 2016, 7, 468-472. [CrossRef]

21. Almansour, A.I.; Kumar, R.S.; Arumugam, R.; Basiri, A.; Kia, Y.; Ali, M.A. An Expedient Synthesis, Acetylcholinesterase Inhibitory Activity, and Molecular Modeling Study of Highly Functionalized Hexahydro-1,6-naphthyridines. BioMed Res. Int. 2015, 2015, 1-9. [CrossRef] [PubMed]

22. Raboisson, P.; Desjarlais, R.L.; Reed, R.; Lattanze, J.; Chaikin, M.; Manthey, C.L.; Tomczuk, B.E.; Marugan, J.J. Identification of novel short chain 4-substituted indoles as potent alphavbeta3 antagonist using structure-based drug design. Eur. J. Med. Chem. 2007, 42, 334. [CrossRef] [PubMed]

23. Huang, X.; Chen, D.; Wu, N.; Zhang, A.; Jia, Z.; Li, X. The synthesis and biological evaluation of a novel series of C7 non-basic substituted fluoroquinolones as antibacterial agents. Bioorg. Med. Chem. Lett. 2009, 19, 4130. [CrossRef] [PubMed]

24. Zeng, L.; Wang, Y.; Kazemi, R.; Xu, S.; Xu, Z.; Sanchez, T.W.; Yang, L.; Debnath, B.; Odde, S.; Xie, H. Repositioning HIV-1 Integrase Inhibitors for Cancer Therapeutics: 1,6-Naphthyridine-7-carboxamide as a Promising Scaffold with Drug-like Properties. J. Med. Chem. 2012, 55, 9492-9509. [CrossRef] [PubMed] 
25. CCDC 1434604 Contains the X-ray Structure Crystallographic Data for This Paper. Available online: http:/ / www.ccdc.cam.ac.uk/conts/retrieving.html (or from the CCDC, 12 Union Road, Cambridge CB2 1EZ, UK; Fax: +44-1223-336033; E-mail: deposit@ccdc.cam.ac.uk).

26. CCDC 1434605 Contains the X-ray Structure Crystallographic Data for This Paper. Available online: http:/ / www.ccdc.cam.ac.uk/conts/retrieving.html (or from the CCDC, 12 Union Road, Cambridge CB2 1EZ, UK; Fax: +44-1223-336033; E-mail: deposit@ccdc.cam.ac.uk).

27. CCDC 1493165 Contains the X-ray Structure Crystallographic Data for This Paper. Available online: http:/ / www.ccdc.cam.ac.uk/conts/retrieving.html (or from the CCDC, 12 Union Road, Cambridge CB2 1EZ, UK; Fax: +44-1223-336033; E-mail: deposit@ccdc.cam.ac.uk).

28. Mittelbach, M. An improved and facile synthesis of 2-Amino-1,1,3-trycyanopropene. Monatsh. Chem. 1985, 116, 689-691. [CrossRef]

Sample Availability: Samples of the compounds 12a-n are available from the authors.

(C) 2017 by the authors. Licensee MDPI, Basel, Switzerland. This article is an open access article distributed under the terms and conditions of the Creative Commons Attribution (CC BY) license (http://creativecommons.org/licenses/by/4.0/). 